\section{More Evolution of the Evidence in Asthma Disease Management-SMART Versus GOAL Clinical Trials Debate the Cost-Benefit of LABA While the Value of Leukotriene Modifiers, Particularly Montelukast, Is Uncertain}

In a previous issue of JMCP, Heaton et al. challenged the value of leukotriene modifiers (LMs) in disease management of asthma. ${ }^{1}$ Based on the 3 clinical outcomes-emergency department (ED) visits, hospitalizations for asthma, and the use of oral prednisone ("steroid burst") to indicate exacerbation of asthma-these authors concluded that LM use was not more effective than nonuse. Worse than no improvement in these 3 clinical outcomes, LM users appeared to have more ED visits, a higher rate of hospitalization, and a higher rate of use of oral prednisone bursts. Heaton et al. calculated that LM use added $\$ 1.63$ per patient per month (PPPM) in costs (in 2002 dollars) for these 3 clinical outcomes compared with LM nonusers diagnosed with asthma.

Coincident to publication of the article by Heaton et al. in JMCP, Martinez suggested, in a commentary published in the New England Journal of Medicine on asthma disease management, that LMs or theophylline might be better alternatives to long-acting beta-agonists (LABAs) in patients not controlled adequately on inhaled corticosteroids (ICSs) alone. ${ }^{2}$ While the motivation for this recommendation was primarily concern regarding the safety risk associated with the LABAs, Mintz, in a follow-up letter, decried overstatement of the risk of LABAs and called this "irresponsible," possibly contributing to suboptimal pharmacotherapy or patient nonadherence. ${ }^{3}$ Mintz argued that a Cochrane Systematic Review published in 2005 found that adding LABAs rather than LMs in the treatment of asthma not controlled by ICSs would prevent 1 exacerbation for every 38 patients treated. This review in the Cochrane Database of Systematic Reviews (Cochrane Database, CD003137) also found LABAs to be superior to LMs in preventing exacerbations requiring systemic steroids, improving lung function and asthma symptoms, and reducing the use of rescue short-acting beta-agonists (SABAs) in adults. ${ }^{4}$

In advocating the use of LABAs over LMs in asthma patients not controlled on ICSs alone, Mintz attempted to dismiss the product label warning and U.S. Food and Drug Administration (FDA) Public Health Advisory for the LABAs regarding the increased chance of a severe asthma episode in LABA users. Mintz argued that the label warning of risk in the use of LABAs is contradicted by results from the Salmeterol Mutlicenter Asthma Research Trial (SMART). The FDA analysis of the SMART study concluded that there was a "small increase in the risk of asthma-related deaths with salmeterol use in the SMART study." The FDA proposed label changes for all salmeterolcontaining products in a letter sent by fax to the manufacturer on September 10, 2004. The present prescribing information in the labels for salmeterol and fluticasone with salmeterol, updated March 2, 2006, includes the following language in the black- box warning, "Long-acting beta 2 -agonists such as salmeterol, one of the active ingredients in ADVAIR DISKUS, may increase the risk of asthma-related death. Therefore, when treating patients with asthma, physicians should only prescribe ADVAIR DISKUS for patients not adequately controlled on other asthmacontroller medications (e.g., low- to medium-dose ICSs) or whose disease severity clearly warrants initiation of treatment with 2 maintenance therapies." 6

Interwoven in the asthma disease management literature and clinical trials is the opportunity for higher rates of improved patient outcomes through more attention to clinical monitoring of titrated dosing of ICSs. The clinical preference for more attention to adequate dosing of ICSs is supported by the GOAL (Gaining Optimal Asthma Control) trial. The focus of this study was to establish the proportion of patients with asthma who could achieve the target level of control with optimal prevention therapy. The study showed that 59\% of patients with previously uncontrolled asthma were well-controlled at 1 year with higher doses of fluticasone alone, while $71 \%$ of patients were well-controlled with higher doses of fluticasone plus LABA. ${ }^{7}$

While the GOAL trial results showed that LABA might have a role as add-on therapy to ICS in asthma patients uncontrolled with ICS alone, the role of LM as add-on therapy to ICS is less certain. A review of 336 citations in the Cochrane Database, including 11 randomized controlled trials (RCTs) performed by Ducharme in 2001 (CD003133), concluded that the addition of an LM to ICS therapy may improve lung function slightly, but this strategy is inferior to increased dosing of ICS. ${ }^{8}$ An update in 2004 from a review of 587 Cochrane Database citations, including 16 RCTs, found the accumulated data from the RCTs insufficient to support the use of LM as a substitute for increased dosing of ICS, and there was no overall value of LM in patients undergoing dose tapering of ICS. ${ }^{9}$

The current asthma management guidelines from the National Asthma Education and Prevention Program (NAEPP) Expert Panel of the National Institutes of Health (National Heart, Lung, and Blood Institute), last updated in 2002, classify asthma into 4 distinct categories. "Step 1" for mild intermittent asthma cases involves no daily medication. ${ }^{10}$ The "Step 2" category for mild persistent cases involves low-dose monotherapy for prevention. It may require revision subsequent to research published last year. Boushey et al. found the group of patients with mild persistent asthma randomized to no controller (ICS) therapy did not have significantly poorer lung function and experienced no greater frequency of asthma exacerbation than those who received regular treatment. ${ }^{11}$ Daily budesonide (ICS) was superior to intermittent budesonide therapy and to daily zafirlukast (LM) therapy in most clinical measures including asthma control and symptom-free days in patients with mild persistent asthma, but daily zafirlukast therapy was not superior to intermittent zafirlukast therapy in any outcome. Based on these and other findings, Boushey et al. estimated that patients 
with mild persistent asthma may require as little as 1 course of inhaled budesonide, on average, every 2 years or oral corticosteroids, on average, once every 8 years. This symptom-driven treatment of mild-to-moderate exacerbations is a radical departure from current guidelines for treatment of mild persistent asthma. ${ }^{12}$ The cost savings from a change in treatment of mild persistent asthma from daily medication use to symptom-driven corticosteroid therapy could be large since up to $75 \%$ of asthma is mild disease. ${ }^{13}$

The 2002 NAEPP asthma management guidelines might also be updated regarding the recommendations for use of LABAs, based on the Cochrane Database review of 18 RCTs performed by Chroinin et al. and first published in October 2004..$^{14}$ The more recent data support the conclusions that (a) combination therapy with ICS and LABA does not increase protection against exacerbations but does improve lung function and asthma symptoms and (b) there is not sufficient evidence to support the initial use of a combination of ICS and LABA over ICS alone. This latter conclusion argues against first-line use of the popular combination of salmeterol and fluticasone (Advair) and provides support for a step-therapy edit imposed by managed care organizations (MCOs).

The clear placement of combination ICS and LABA in only "Step 3" (moderate persistent) and "Step 4" (severe persistent asthma) of the NAEPP guidelines and the Cochrane Database review by Chroinin appear to be at odds with the popularity and sales of Advair. Advair sales in community pharmacies increased by $21.8 \%$ in 2005 to $\$ 2.83$ billion, making it the fifth-highestexpenditure drug in the United States. ${ }^{15}$ For the first 3 months of 2006, Advair was the fourth-highest-expenditure drug in one PBM database, with an average allowed cost of $\$ 5.25$ per day or $\$ 157$ per 30-day supply. ${ }^{16}$ Worldwide sales of Advair were $\$ 5.6$ billion in 2005 , up $19 \%$, making it the fourth-highest-selling drug in the world. Advair is also one of the most heavily advertised drugs, with \$137 million in advertising, and Wall Street analysts have suggested that the sales figures prove that the drug is no longer used in the narrow population of severe asthma patients. ${ }^{17}$

While the LABAs appear to have a minor role in asthma disease management, based on the clinical evidence of cost versus benefit, the LM agents should play an even smaller role and be used as only second-line treatment in asthma disease management. The current (2002) version of the NAEPP asthma management guidelines clearly puts LMs in a minor role. The use of LMs is recommended only for one category of patients, those in "Step 3" with moderate persistent asthma and then only as "alternative treatment" to the "preferred treatment" consisting of low-to-medium-dose ICS + LABA. These asthma treatment guidelines also do not give preference to (a) the use of either theophylline or LM with low-to-medium-dose ICS or (b) an increase in ICS monotherapy into the medium-dose range.

In a crossover study of 20 adults with persistent asthma requiring at least $400 \mathrm{mcg}$ per day of an inhaled steroid, Wilson et al. showed that pulmonary function tests were improved similarly with montelukast or salmeterol. However, salmeterol was superior to montelukast because it required less use of SABA rescue therapy and had better asthma symptom scores. ${ }^{18}$ Fish et al., in a study of 948 asthma patients not controlled with ICS, found that salmeterol $50 \mathrm{mcg}$ twice daily was superior to oral montelukast $10 \mathrm{mg}$ daily. ${ }^{19}$ For only 1 outcome measure, likelihood of an asthma exacerbation, were the 2 drugs similar over 3 months of therapy. For the 6 other clinical outcome measures-daytime symptoms, percentage of symptom-free days, percentage of rescue-free days, albuterol use, nighttime awakenings, and patient dissatisfaction with how well and how fast the 2 drugs worked-salmeterol was superior to montelukast.

Critecos et al. found potential risk in the use of the LM zafirlukast in older adults in a retrospective analysis of 5 randomized, double-blind, double-dummy studies 4 to 12 weeks in duration of 1,742 patients younger than 50 years and 243 patients aged 50 years or older. ${ }^{20}$ Their analyses indicate that inhaled fluticasone controls inflammation effectively in older patients, but zafirlukast did not provide either adequate bronchodilatation or the anti-inflammatory activity necessary to achieve effective asthma control in asthma patients older than 50 years. Critecos et al. suggested that the LM may mask inflammation.

In this issue of JMCP, Allen-Ramey et al. conducted an analysis of administrative claims incurred prior to the market introduction of combination salmeterol-fluticasone in an effort to show that dual therapy with montelukast + ICS was similar or better in utilization and cost outcomes compared with dual therapy with salmeterol + ICS. ${ }^{21}$ In an accompanying commentary, Crownover points out some of the methodological shortcomings in the analyses by Allen-Ramey et al., including the failure to use the 4-level disease proxy to construct their propensity score (matching) model, which, if used, might have excluded $75 \%$ of the cases in that study since only those patients in Step 3 (moderate persistent asthma) or higher generally should be considered for add-on therapy with montelukast or salmeterol. ${ }^{22}$

Allen-Ramey et al. found comparable utilization of rescue medication (oral corticosteroids) for users of combination ICS + montelukast and users of combination ICS + salmeterol and slightly lower odds of ED visits or hospitalization for ICS + montelukast (odds ratio [OR], 0.58; 95\% confidence interval (CI), 0.35-0.98). Of perhaps more clinical significance was the higher rate of use of SABAs such as albuterol among the ICS + montelukast patients (RR [relative risk] compared with ICS + salmeterol, 1.33, 95\% CI, 1.17-1.52). Previously, Stempel et al. claimed that experimental clinical studies demonstrate that the addition of salmeterol to ICS is superior to the addition of montelukast to ICS and found in their administrative claims analysis that patients in the ICS + salmeterol group had 35\% fewer SABA claims compared with patients in the ICS + montelukast group. ${ }^{23}$ This higher use of SABAs among ICS + montelukast patients is very similar to the $38 \%$ higher use 
of SABAs found among ICS + montelukast patients in the study by Allen-Ramey et al. (Table 3, page 314), an average of 4.36 pharmacy claims for SABAs in the postindex period for ICS + montelukast versus 3.16 for ICS + salmeterol.

It is also noteworthy that Stempel et al. found 63\% higher total adjusted asthma costs for patients who received ICS + montelukast (compared with ICS + salmeterol), which is consistent with the finding of Heaton et al. of an additional \$1.63 PPPM in costs (in 2002 dollars) for LM users diagnosed with asthma compared with nonusers of LMs diagnosed with asthma. ${ }^{1}$

Montelukast is on the radar screen of every managed care pharmacist with responsibility for drug benefit costs and determining the value of alternate drug therapy. ${ }^{24}$ Montelukast had community pharmacy sales of $\$ 1.85$ billion in 2004 , placing it at rank number 14 by expenditure among all brand-name drugs. ${ }^{25}$ Community pharmacy sales increased by $13 \%$ in 2005 to $\$ 2.09$ billion, making montelukast the ninth-highest-ranked drug in total prescription drug sales. ${ }^{15}$ Monelukast was used by 50 times more patients than zafirlukast (Accolate), the other LM, and had a discounted allowed charge per day of \$3.09 before copayment, or $\$ 93$ per 30 -day supply in the first 3 months of 2006. ${ }^{16}$ Some of this spending on montelukast is for allergic rhinitis, and Lakomski and Chitre found, in a previous issue of JMCP, that even years prior to the FDA-approved indication for allergic rhinitis, 25\% of LM utilization in 2001-2002 was not for asthma. ${ }^{26}$ Even at $75 \%$ of current spending on montelukast, the drug ranks in the top 10 drugs in the United States, second only to combination fluticasone-salmeterol (Advair, rank \#5) among the high-expenditure drugs used in treating asthma.

The return on investment for this tremendous spending on montelukast should be of great interest. The Chronic Asthma Protocol 049 in the original FDA drug application for montelukast showed a $4.65 \%$ improvement in forced expiratory volume in 1 second ( $\left[\mathrm{FEV}_{1}\right]$ : $8.71 \%$ for montelukast vs. $4.16 \%$ for placebo) over 8 weeks of treatment with montelukast in 198 patients versus 133 patients who received placebo. ${ }^{27} \mathrm{~A}$ more interesting finding was that for patients who received placebo, the mean percentage of days over the 8 weeks with an asthma exacerbation was $25.67 \%$ versus $20.58 \%$ for montelukast $(P=0.049)$. Headache determined to be drug-related occurred more often in the montelukast group, $3.5 \%$ vs. $0.7 \%$ for placebo.

A meta-analysis of 1 pediatric and 12 adult clinical trials for the primary outcome of the number of exacerbations requiring systemic glucocorticoids found that patients treated with LMs were $60 \%$ more likely to require systemic glucocorticoid as a result of exacerbation of asthma symptoms compared with patients on monotherapy with an ICS. ${ }^{28}$ LMs were also more likely to be withdrawn as a result of inadequate asthma control (RR, 2.5). The study concluded that $400 \mathrm{mcg}$ of beclomethasone or $200 \mathrm{mcg}$ of fluticasone is more effective than $10 \mathrm{mg}$ per day of montelukast or $20 \mathrm{mg}$ of zafirlukast twice daily.

The hope for some potential value of montelukast in a subset of asthma patients with allergic rhinitis was undercut by The Medical Letter consultants who concluded in October 2005 that comparative studies with oral antihistamines and intranasal steroids are necessary, particularly since these 2 categories of therapeutic alternatives cost (much) less than montelukast. ${ }^{29}$ The current guidelines for the step-wise approach to pharmacotherapy for seasonal allergic rhinitis from the American Academy of Allergy, Asthma \& Immunology do not mention LM. ${ }^{30}$

The evidence of the value of montelukast in patients with a history of both allergic rhinitis and asthma is confined to a single randomized, crossover, placebo-controlled study in 52 patients with symptoms provoked by exposure to cats. ${ }^{31}$ In a large study of nearly 900 patients, montelukast $10 \mathrm{mg}$ was found to be inferior to fluticasone nasal spray $200 \mathrm{mcg}$ per day in controlling symptoms of allergic rhinitis in patients with persistent asthma. ${ }^{32}$ Fluticasone nasal spray became available by generic name in the first week in April 2006, ${ }^{33}$ and the MCO cost will soon be $\$ 1.00$ or less per day of therapy. So, where is the value of montelukast in the treatment of either a small subset of asthma patients or in perhaps an even smaller subset of allergy patients when fluticasone nasal spray is more effective and will soon cost less than one third the price of montelukast, and the cost of loratadine over-the-counter (OTC, Claritin) is less than $\$ 1.00$ per day of therapy?

Perhaps elsewhere are some data to justify the direct drug cost of more than $\$ 3$ per day for montelukast for the treatment of perennial allergic rhinitis, the second indication for this drug approved by the FDA in July 2005..$^{34}$ The most recent study on this subject found that $10 \mathrm{mg}$ per day of montelukast was no more effective than $240 \mathrm{mg}$ per day of OTC pseudoephedrine in adult patients with ragweed allergic rhinitis documented by (a) positive skin test to ragweed and (b) history of symptoms during previous ragweed seasons. There was no difference in the outcome measures of nasal peak inspiratory flow and diurnal and nocturnal rhinoconjunctivitis quality-of-life scores. ${ }^{35}$ Not only was there no difference between these 2 drugs in 3 measures of effectiveness, pseudoephedrine was superior to montelukast in the symptoms of nasal congestion, and no difference was found between the 2 drugs in tolerability.

The major difference, of course, between montelukast and OTC pseudoephedrine or OTC loratadine is its much higher cost. ${ }^{36}$ It is possible to use OTC pseudoephedrine or OTC loratadine to treat the symptoms of allergic rhinitis, more effectively, in 4 patients for the same cost as treating one patient with montelukast, before consideration of the cost of physician office visits to obtain the prescription drug montelukast. This creates a terrific opportunity for the use of a step-therapy protocol for either indication for montelukast, asthma or allergy, particularly since montelukast was the eleventh-highest-volume brandname prescription drug in dispensed units in $2005^{37}$ and the 
ninth-highest-expenditure drug in total community pharmacy sales in 2005.

\section{Frederic R. Curtiss, PhD, RPh, CEBS}

Editor-in-Chief

fcurtiss@amcp.org

\section{REFERENCES}

1. Heaton PC, Guo JJ, Horrnung RW, et al. Analysis of the effectiveness and cost benefit of leukotrience modifiers in adults with asthma in the Ohio Medicaid population. J Manag Care Pharm. 2006;12(1):33-42.

2. Martinez FD. Safety of long-acting beta-agonists—an urgent need to clear the air. N Engl J Med. 2005;353:2637-39.

3. Mintz ML. Safety of long-acting beta-agonists. N Engl J Med. 2006;354: 1206-07.

4. Ram FS, Cates CJ, Ducharme FM. Long-acting beta2-agonists versus antileukotrienes as add-on therapy to inhaled corticosteroid for chronic asthma. Cochrane Database Syst Rev. 2005;(1):CD003137. Available at:

http://www.mrw.interscience.wiley.com/cochrane/clsysrev/articles/CD003137/ frame.html. Accessed April 26, 2006.

5. U.S. Food and Drug Administration. Medical officer review. Salmeterol Postmarketing Study Review (SMART Study). Available at: http://www.fda.gov/ ohrms/dockets/ac/05/briefing/2005-4148B1_03_02-FDA-Smart-Study.pdf. Accessed April 2, 2006.

6. U.S. Food and Drug Administration. Serevent and Advair Diskus labels. Updated March 2, 2006. Available at: http://www.fda.gov/cder/drug/infopage/ LABA/default.htm. Accessed May 1, 2006.

7. Bateman ED, Boushey HA, Bousquet J, et al. Can guideline-defined asthma control be achieved? The Gaining Optimal Asthma ControL study. Am J Respir Crit Care Med. 2004;170(8):836-44.

8. Ducharme F. Addition of anti-leukotriene agents to inhaled corticosteroids for chronic asthma. Cochrane Database Syst Rev. 2001;3:CD003133.

9. Ducharme F, Schwartz Z, Hicks G, Kakuma R. Addition of anti-leukotriene agents to inhaled corticosteroids for chronic asthma. Cochrane Database Syst Rev. 2004;1:CD003133. Available at: http://www.mrw.interscience.wiley.com/ cochrane/clsysrev/articles/CD003133/frame.html. Accessed April 26, 2006.

10. National Asthma Education and Prevention Program. Expert Panel Report: guidelines for the diagnosis and management of asthma - update on selected topics 2002. Available at: http://www.nhlbi.nih.gov/guidelines/asthma/ index.htm. Accessed April 2, 2006.

11. Boushey HA, Sorkness CA, King TS, et al. for the National Heart, Lung, and Blood Institute's Asthma Clinical Research Network. N Engl J Med. 2005;352:1519-28.

12. Fabbri LM. Does mild persistent asthma require regular treatment? N Engl J Med. 2005;352:1589-91.

13. Auerbach I, Springer C, Godfrey S. Total population survey of the frequency and severity of asthma in 17 year old boys in an urban area of Israel. Thorax. 1993;48:139-41.

14. Chroinin NM, Greenstone IR, Ducharme FM. Addition of inhaled longacting betaz-agonists to inhaled steroid as first line therapy for persistent asthma in steroid-naive adults. Cochrane Database Syst Rev. 2004:4:005307. Available at: http://www.mrw.interscience.wiley.com/cochrane/clsysrev/articles/CD005307/ frame.html. Accessed April 26, 2006.

15. Top 200 brand-name drugs by retail dollars in 2005. Drug Top. March 6, 2006:26.

16. Data search performed April 6, 2006, of the data warehouse of a national pharmacy benefits manager representing approximately 500,000 beneficiaries of small employer drug benefit plans for pharmacy claims with dates of service from January 1, 2006, through March 31, 2006.
17. Langreth R. Trouble breathing? Forbes. April 6, 2006. Available at: http://www.forbes.com/technology/2006/04/05/cz_rl_advair_magp80.html. Accessed May 1, 2006.

18. Wilson AM, Dempsey OJ, Sims EJ, Lipworth BJ. Evaluation of salmeterol of montelukast as second-line therapy for asthma not controlled with inhaled corticosteroids. Chest. 2001;119:1021-26.

19. Fish JE, Israel E, Murray JJ, et al. Salmeterol powder provides significantly better benefit than montelukast in asthmatic patients receiving concomitant inhaled corticosteroid therapy. Chest. 2001;120:423-30.

20. Critecos P, Knobil K, Edwards LD, Rickard KA, Dorinsky P. Loss of response to treatment with leukotriene receptor antagonists but not inhaled corticosteroids in patients over 50 years of age. Ann Allergy Asthma Immunol. 2002;88(40:401-09

21. Alley-Ramey F, Bukstein D, Luskin A, Sajjan S, Marson LE. Administrative claims analysis of asthma-related heatlh care utilization for patients who received inhaled corticosteroids with either montelukast or salmeterol as combination therapy. J Manag Care Pharm. 2006;12(4):310-21

22. Crownover BC. PP-ICONS-another tool to help interpret asthma utilization studies. J Manag Care Pharm. 2006;12(4):341-42.

23. Stempel DA, O'Donnell JC, Meyer JW. Inhaled corticosteroids plus salmeterol or montelukast: effects on resource utilization and costs. J Allergy Clin Immunol. 2002;109(3):433-39.

24. Curtiss FR. Asthma disease management-evidence-based medicine must be dynamic. J Manag Care Pharm. 2006;12(1):80-82.

25. Data from Verispan. Top 200 brand-name drugs by retail dollars in 2004 Drug Top. February 21, 2005:18.

26. Lakomski PG, Chitre M. Evaluation of the utilization patterns of leukotriene modifiers in a large managed care health plan. J Manag Care Pharm. 2004;10(2):115-21.

27. FDA Center for Drug Evaluation and Research. Application \#20-830, medical reviews. Medical officer review, January 20, 1998. Available at: http://www.fda.gov/cder/foi/nda/98/20830_Singulair_medr.pdf. Accessed December 18, 2005.

28. Ducharme FM. Inhaled glucocorticoids versus leukotriene receptor antagonists as single agent asthma treatment: systematic review of current evidence. BMJ. 2003;326:621-25.

29. Montelukast (Singulair) for perennial allergic rhinitis. Med Letter. 2005;47 (1220):87-88

30. American Academy of Allergy, Asthma \& Immunology. Stepwise approach to pharmacotherapy for seasonal allergic rhinitis. The Allergy Report. Available at: http://www.theallergyreport.com/professional/ rhinitis0.html. Accessed May $1,2006$.

31. Perry TT, Corren J, Phillip G, et al. Protective effect of montelukast on lower and upper respiratory tract responses to short-term cat allergen exposure Ann Allergy Asthma Immunol. 2004;93(5):431-38

32. Nathan RA, Yancey SW, Waitkus-Edwards K, et al. Fluticasone propionate nasal spray is superior to montelukast for allergic rhinitis while neither affects overall asthma control. Chest. 2005;128(4):1910-20.

33. Par Pharmaceutical resumes shipment of generic Flonase. NewsEdge. April 6, 2006.

34. U.S. Food and Drug Administration. Letter for final printed labeling (FPL) for supplements NDA 20-829/S-033, NDA 20-830/S-035, NDA 21-409/S-012. July 27, 2005. Available at: http://www.fda.gov/cder/foi/appletter/2005/020829s033, 020830s035,021409s012ltr.pdf. Accessed April 28, 2006.

35. Mucha SM, deTineo M, Naclerio RM, Baroody FM. Comparison of montelukast and pseudoephedrine in the treatment of allergic rhinitis. Arch Otolaryngol Head Neck Surg. 2006;132:164-72.

36. Available at: www.drugstore.com for montelukast (Singulair) $10 \mathrm{mg}$ oral tablet and pseudoephedrine $240 \mathrm{mg}$ (Sudafed 24 Hour). Accessed April 28, 2006.

37. Top 200 brand-name drugs by units in 2005. Drug Top. March 20, 2006:25. 